\section{Adoption of robotic surgery: driven by market competition or a desire to improve patient care?}

\section{Authors' reply}

In our paper, ${ }^{1}$ we describe how competitive forces have strongly influenced the configuration of prostate cancer services, leading to the closure of one in four of the centres providing prostate cancer surgery in the National Health Service (NHS) in England.

From our analysis, patients were more likely to travel to hospitals that provided robotic surgery, frequently bypassing their local service if this technology was not available. Sean Fletcher and colleagues indicate that they disagree with our assertion that the rapid adoption of robotic surgery took place in the absence of evidence that it produced better outcomes. They point out that an observational study, published in $2012,{ }^{2}$ reported shorter hospital stay, fewer perioperative complications, and a lower blood transfusion rate in patients who had a robot-assisted prostatectomy than in those who had open surgery.

However, we feel that it is unlikely this publication had an impact on the adoption of robotic surgery in the NHS in England during our study period (2010-014), because there is a considerable lag time between the decision to purchase robotic equipment and the ability to provide robotic surgery. Additionally, the rapid integration of robotic surgery was already continuing apace at the time these data were published. Finally, there was-and still is-a dearth of robust evidence on the effectiveness of robotic surgery on long-term functional outcomes and cancer cure. $^{3.4}$

We do not view competition in a negative light. However, we do feel that its effect on the ability of health services to provide equitable, affordable, high-quality care should be carefully scrutinised.

We declare no competing interests.

*Ajay Aggarwal, Daniel Lewis, Malcolm Mason, Arnie Purushotham, Richard Sullivan, Jan van der Meulen ajay.aggarwal@Ishtm.ac.uk

Department of Health Services Research \& Policy (AA, JvdM) and Department of Social and Environment Health Research (DL), London School of Hygiene and Tropical Medicine, London WC1H 9SH, UK; Clinical Effectiveness Unit, Royal College of Surgeons of England, London, UK (AA, JvdM); Department of Social and Environment Health Research, London School of Hygiene and Tropical Medicine, London, UK; School of Medicine, Cardiff University, Cardiff, UK (MM); Division of Cancer Studies, Kings College London, London, UK (AP); and Institute of Cancer Policy, King's College London, London, UK (RS)

1 Aggarwal A, Lewis D, Mason M, Purushotham A, Sullivan R, van der Meulen J. Effect of patient choice and hospital competition on service configuration and technology adoption within cancer surgery: a national, populationbased study. Lancet Oncol 2017; 18: 1445-53.

2 Trinh QD, Sammon J, Sun M, et al. Perioperative outcomes of robot-assisted radical prostatectomy compared with open radical prostatectomy: results from the nationwide inpatient sample. Eur Urol 2012; 61: 679-85.

3 Ramsay C, Pickard R, Robertson C, et al. Systematic review and economic modelling of the relative clinical benefit and costeffectiveness of laparoscopic surgery and robotic surgery for removal of the prostate in men with localised prostate cancer. Health Technol Assess 2012; 16: 1-313.

4 Ilic D, Evans SM, Allan CA, Jung JH, Murphy D, Frydenberg M. Laparoscopic and roboticassisted versus open radical prostatectomy for the treatment of localised prostate cancer. Cochrane Database Syst Rev 2017; 9: Cd009625. 